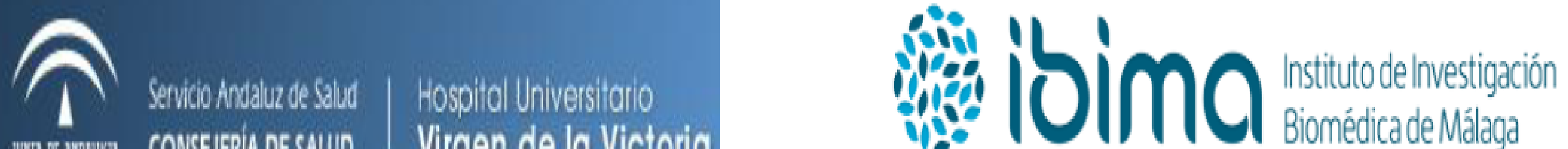

\section{Extra virgin olive oil and red wine polyphenols modulate fecal microbiota and reduce metabolic risk factors in high insulin resistant obese patients}

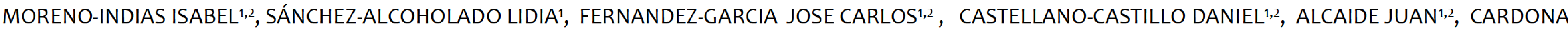
FERNANDO',2,QUEIPO-ORTUÑO MARÍA ISABEL ${ }^{1,2}$

${ }^{1}$ Clinical Management Unit of Endocrinology and Nutrition of the Virgen de la Victoria Hospital, Biomedical Research Institute of Malaga (IBIMA), Malaga, Spain 2Biomedical Research Networking Center for Pathophysiology of Obesity and Nutrition, CIBERobn, Madrid, Spain

INTRODUCTION

$\checkmark$ Beneficial effects of prebiotic food products on energy homoeostasis, satiety regulation, body weight gain and change in the composition of the gut microbiota have been analyzed, supporting the hypothesis that the gut microbiota composition may contribute to the modulation of metabolic processes associated with insulin resistance.

$\checkmark$ Protective effects of polyphenols such as the modulation of vascular and platelet function, blood pressure, and an improved plasma lipid profile has been tested.

$\checkmark$ The use of polyphenols may be a potential mechanism for prevention of cardiovascular and metabolic alterations associated with obesity.

\section{OBJECTIVE}

This study evaluated the prebiotic effect of a moderate intake of extra virgin olive oil (Oov) (50 g/dayl) and red wine (Rw) (270 ml /day) polyphenols on modulating the gut microbiota composition and the improvement of the metabolic risk factors in obese subjects with high insulin resistance.

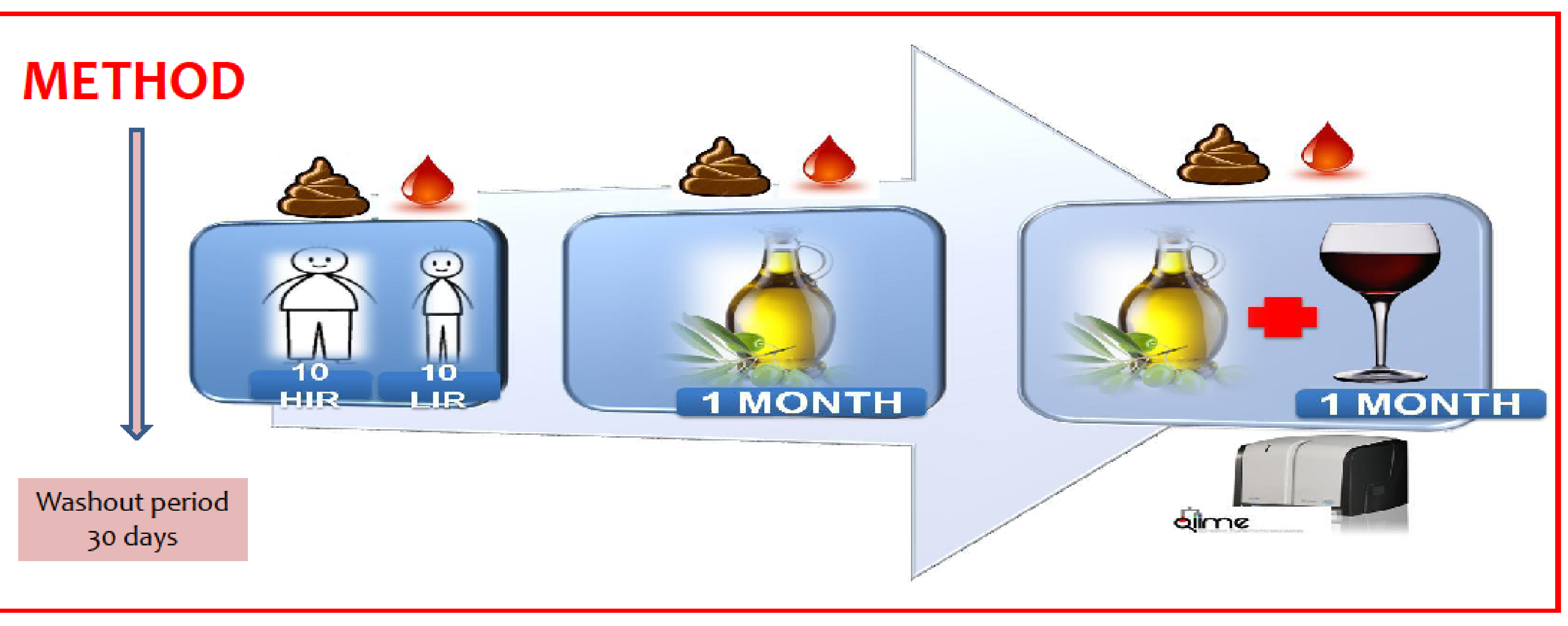

Figure 1. PCoA analysis of bacterial communities from fecal samples subjected to 454 sequencing. Results are based on unweighted Unifrac metrics.
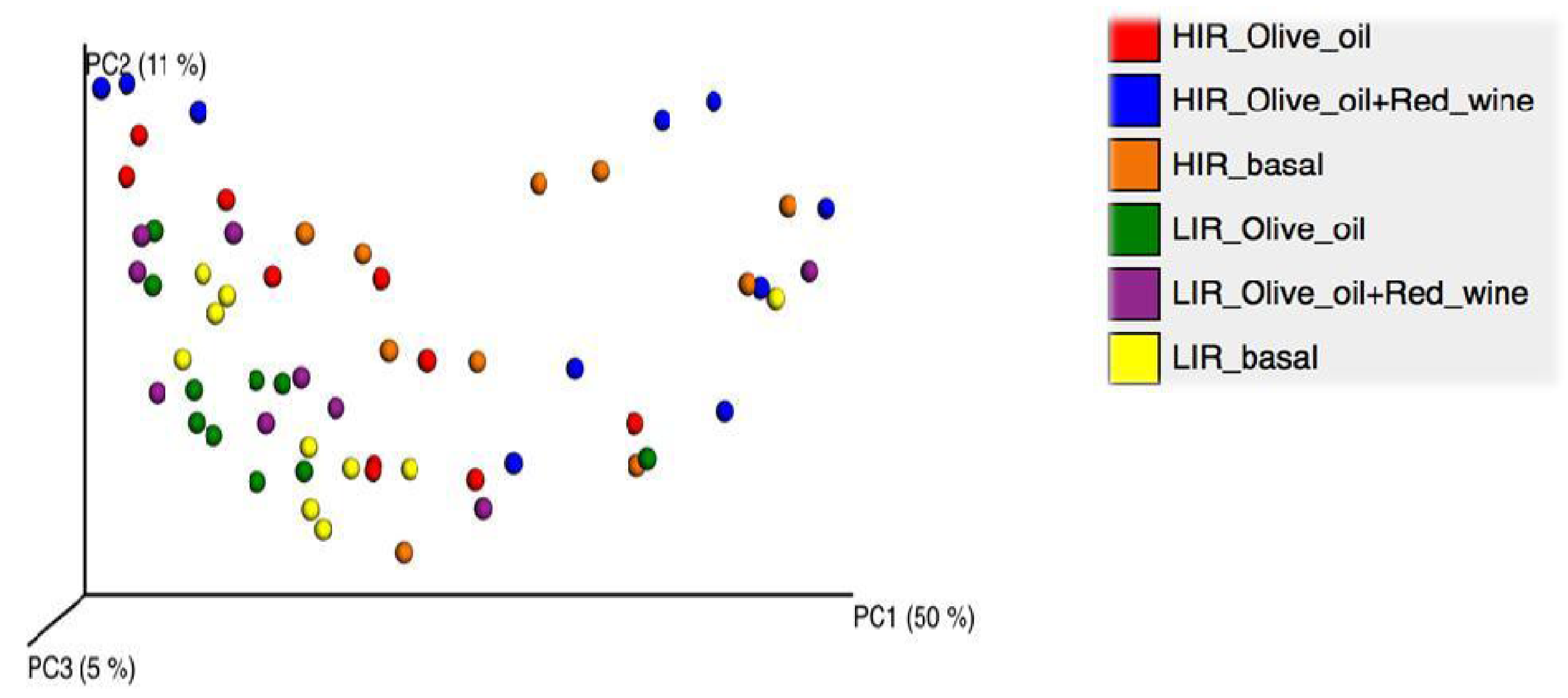

Figure 2. Pyrosequencing analysis of phyla in the LIR and HIR groups after the different intake periods.

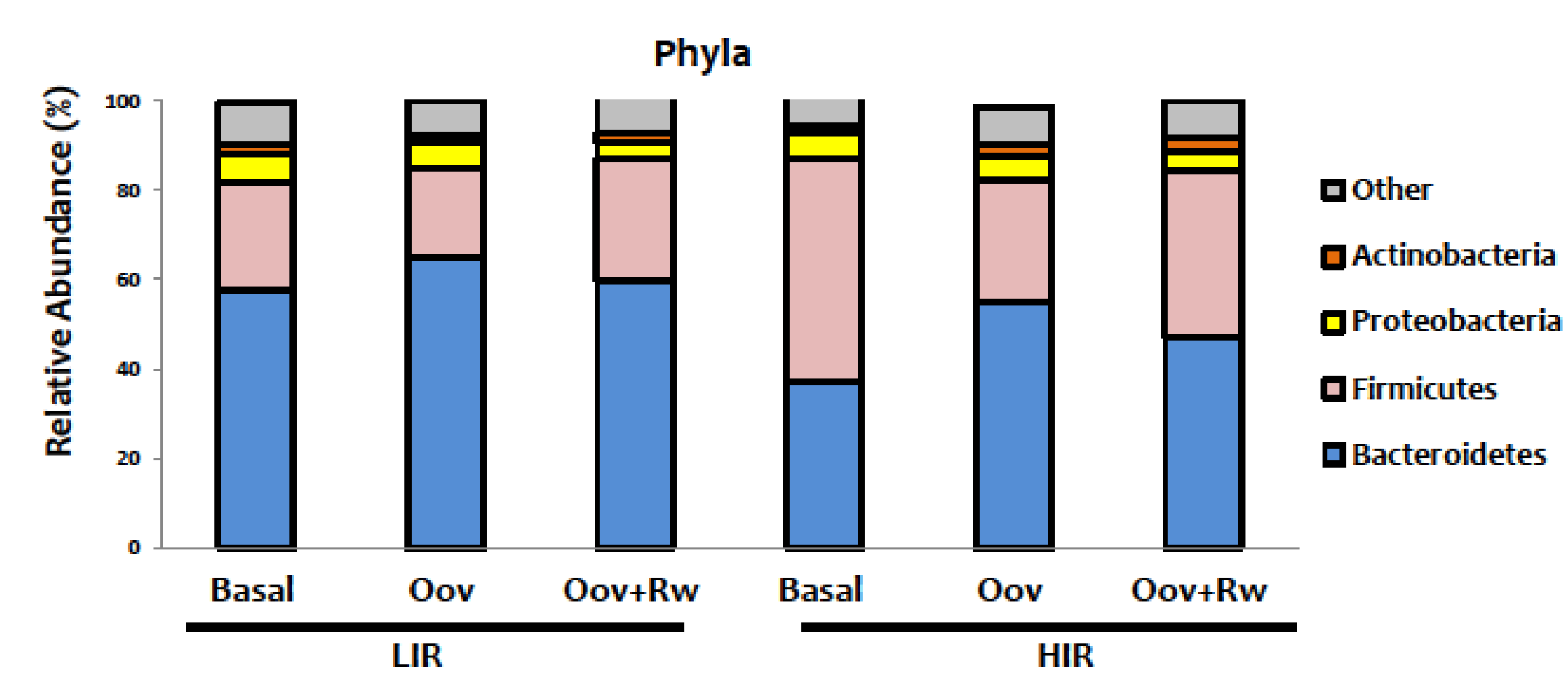

RESULTS

\begin{tabular}{|c|c|c|c|c|c|c|}
\hline & & $\operatorname{LIR}(\mathrm{n}=10)$ & & & HIR (n=10) & \\
\hline & BASAL & & Oov $+\mathbf{R w}$ & BASAL & Oov & $\mathbf{O o V}+\mathbf{R w}$ \\
\hline BMI (kg/m2) & $25.37 \pm 2.40^{0^{a^{*}}}$ & $24.86 \pm 2.37^{\mathrm{as}}$ & $24.61 \pm 2.49^{\text {as }}$ & $38.82 \pm 2.58^{\mathrm{a}}$ & $37.81 \pm 2.22^{\mathrm{a}}$ & $37.61 \pm 2.29^{\mathrm{a}}$ \\
\hline Waist (cm) & $93.39 \pm 6.35^{a^{a^{*}}}$ & $92.70 \pm 6.87^{\text {as }}$ & $92.23 \pm 7.17^{\text {as }}$ & $123.10 \pm 15.39^{\mathrm{a}}$ & $122.05 \pm 13.39^{\mathrm{a}}$ & $121.80 \pm 13.89^{\circ}$ \\
\hline Hip (cm) & $104.8 \pm 4.94^{a^{*}}$ & $103.80 \pm 4.33^{3 \mathrm{~s}}$ & $103.00 \pm 5.22^{25}$ & $128.25 \pm 18.50^{\mathrm{a}}$ & $127.32 \pm 15.98^{\mathrm{a}}$ & $127.67 \pm 14.49^{\circ}$ \\
\hline DBP (mmHg) & $64.80 \pm 10.19^{\mathrm{a} *}$ & $63.40 \pm 11.62^{a}$ & $63.90 \pm 11.24^{a}$ & $76.80 \pm 16.94^{\mathrm{a}}$ & $73.0 \pm 16.66^{\mathrm{a}}$ & $72.10 \pm 17.59^{\mathrm{a}}$ \\
\hline SBP (mmHg) & $114.70 \pm 9,28^{a^{a}}$ & $112.70 \pm 12.33^{\mathrm{a}}$ & $111.20 \pm 8.28^{\mathrm{a}}$ & $129.20 \pm 18.51^{\mathrm{a}}$ & $123.3 \pm 15.99^{\mathrm{a}}$ & $122.0 \pm 17.62^{a}$ \\
\hline Glucose (mg/dL) & $88.40 \pm 5.97^{\mathrm{a}^{*}}$ & $86.10 \pm 6.69^{\text {as }}$ & $86.65 \pm 6.14^{\text {as }}$ & $137.70 \pm 10.05^{\mathrm{a}}$ & $102.90 \pm 8.43^{b}$ & $100.5 \pm 8.95^{b}$ \\
\hline Insulin (mg/dl) & $9.05 \pm 1.55^{a *}$ & $6.57 \pm 1.38^{b 5}$ & $6.68 \pm 1.39^{6 \xi}$ & $23.78 \pm 3.24^{\mathrm{a}}$ & $15.96 \pm 2.45^{\mathrm{b}}$ & $16.83 \pm 2.80^{\mathrm{b}}$ \\
\hline Uric acid (mg/dL) & $4.43 \pm 1.27^{\mathrm{a} *}$ & $4.40 \pm 1.50^{\mathrm{a}}$ & $4 \cdot 55 \pm 1.31^{\mathrm{a}}$ & $5.76 \pm 1.39^{a}$ & $5.69 \pm 1.22^{\mathrm{a}}$ & $6.11 \pm 1.53^{\mathrm{a}}$ \\
\hline $\begin{array}{l}\text { Triglycerides } \\
\text { (mg/dL) } \\
\text { Cholesterol }\end{array}$ & $101.40 \pm 20.22^{\mathrm{a} *}$ & $96.0 \pm 28.18^{a \mathrm{~s}}$ & $93.50 \pm 21.2^{25}$ & $219.90 \pm 23.96^{a}$ & $174.10 \pm 26.13^{b}$ & $179.20 \pm 24.25^{\mathrm{b}}$ \\
\hline $\begin{array}{l}\text { (mg/dL) } \\
\text { LDL cholesterol }\end{array}$ & $177.70 \pm 17.43^{\mathrm{a} *}$ & $166.5 \pm 16.18^{\mathrm{a}}$ & $169.60 \pm 15.29^{a}$ & $224.30 \pm 14.18^{a}$ & $178.30 \pm 16.43^{b}$ & $175.00 \pm 12.15^{b}$ \\
\hline $\begin{array}{l}(\mathbf{m g} / \mathbf{d L}) \\
\text { HDLcholesterol }\end{array}$ & $119.00 \pm 22.85^{\mathrm{a}}$ & $109.86 \pm 21.55^{a}$ & $106.06 \pm 20.86^{a}$ & $120.26 \pm 37.36^{a}$ & $113 \cdot 30 \pm 34 \cdot 30^{a}$ & $111.18 \pm 29.69^{a}$ \\
\hline $\begin{array}{l}\text { (mg/dL) } \\
\text { CRP (mg/dL) }\end{array}$ & $\begin{array}{c}65.70 \pm 15 \cdot 50^{\mathrm{a} *} \\
4.89 \pm 1.10^{\mathrm{a} *}\end{array}$ & $\begin{array}{c}67.40 \pm 11.56^{\mathrm{a}} \\
3.14 \pm 1.58^{\mathrm{b}}\end{array}$ & $\begin{array}{c}66.40 \pm 12.29^{\mathrm{a}} \\
3.87 \pm 0.76^{\mathrm{b}}\end{array}$ & $\begin{array}{c}41.60 \pm 11.50^{\mathrm{a}} \\
8.71 \pm 5.40^{\mathrm{a}}\end{array}$ & $\begin{array}{c}52.40 \pm 13.34^{\mathrm{b}} \\
5.43 \pm 1.83^{\mathrm{b}}\end{array}$ & $\begin{aligned} 53.40 & \pm 13.05^{\mathrm{b}} \\
5.28 & \pm 1.55^{\mathrm{b}}\end{aligned}$ \\
\hline
\end{tabular}

Figure 2. Analysis of the diversity and similarity of microbial communities in both study groups

\begin{tabular}{|l|c|c|c|c|c|c|}
\hline & \multicolumn{3}{|c|}{ HIR } & \multicolumn{3}{c|}{ LIR } \\
\hline & Basal & Oov & Oov+Rw & Basal & Oov & Oov+Rw \\
\hline Chao 1 & $169.88 \pm 56.4$ & $164.54 \pm 42.5$ & $157.38 \pm 27.5$ & $168.41 \pm 45.1$ & $162.28 \pm 32.9$ & $159.66 \pm 41.2$ \\
Shannon & $5.16 \pm 0.31$ & $5.16 \pm 0.59$ & $4.33 \pm 0.80$ & $5.15 \pm 0.58$ & $5.09 \pm 0.50$ & $4.71 \pm 0.37$ \\
\hline
\end{tabular}

\section{CONCLUSIONS}

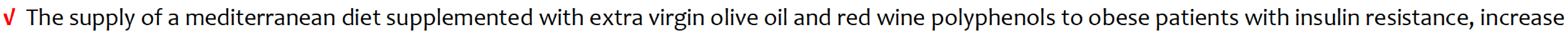
HDL levels and decrease triglyceride and CRP levels, improving the cardiovascular risk associated with these patients.

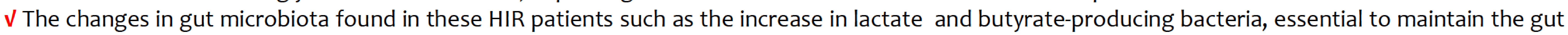
integrity and the decrease in mucin-degrading bacteria, could be responsible for the improvement observed in these metabolic markers.

This study has been supported by the grants from Instituto de Salud Carlos III; CP13/00065; CPII13/00023 and co-founded by Fondo Europeo de Desarrollo Regional - FEDER. contact: maribelqo@gmail.com

REFERENCES

-Queipo-Ortuño, M. I., Boto-Ordóñez, M., Murri, M., Gomez-Zumaquero, J. M., et al., Influence of red wine polyphenols and ethanol on the gut microbiota ecology and biochemical biomarkers. Am. J. Clin. Nutr.2012, 95, 1323-1334. -Cardona, F., Andrés-Lacueva, C., Tulipani, S., Tinahones, F. J., Queipo-Ortuño M.I. Benefits of polyphenols on gut microbiota and implications in human health. J. Nutr. Biochem. 2013, 24, 1415-1422.

-Boto-Ordóñez, M., Urpi-Sarda, M., Queipo-Ortuño, M. I., et al., High levels of Bifidobacteria are associated with increased levels of anthocyanin microbial metabolites: a randomized clinical trial. Food Funct. 2014, 5, 1932-1938.

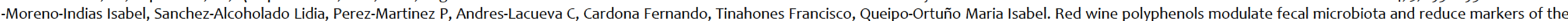
metabolic syndrome in obese patients. Food \& Function 2016 Apr 20;7(4):1775-87

Topic: Obesity

Figure 3. Family-level microbial microbial classification of bacteria from LIR and HIR stool samples during the study.

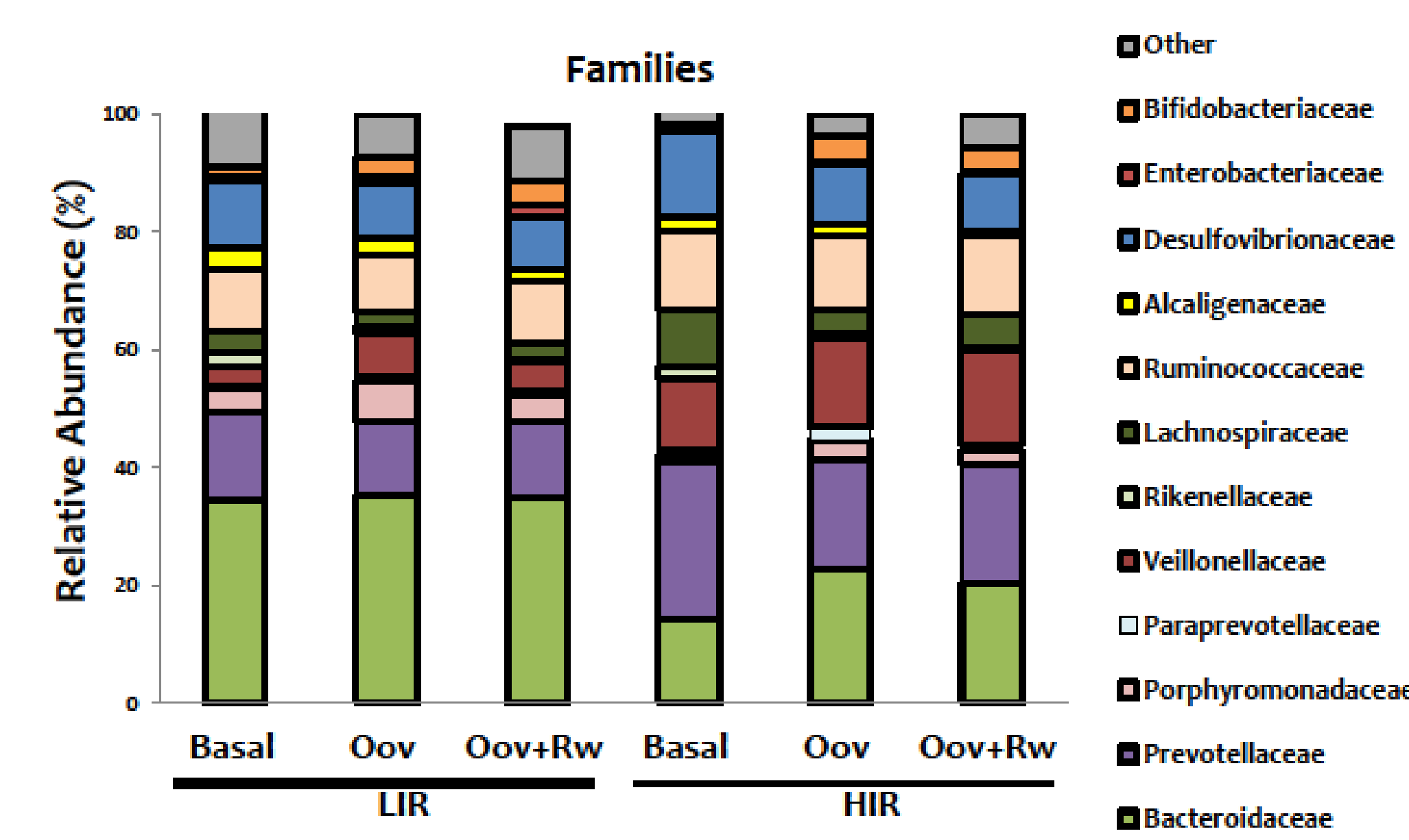

Fure 4. Relative abundance of bacterial genera in the microbiot

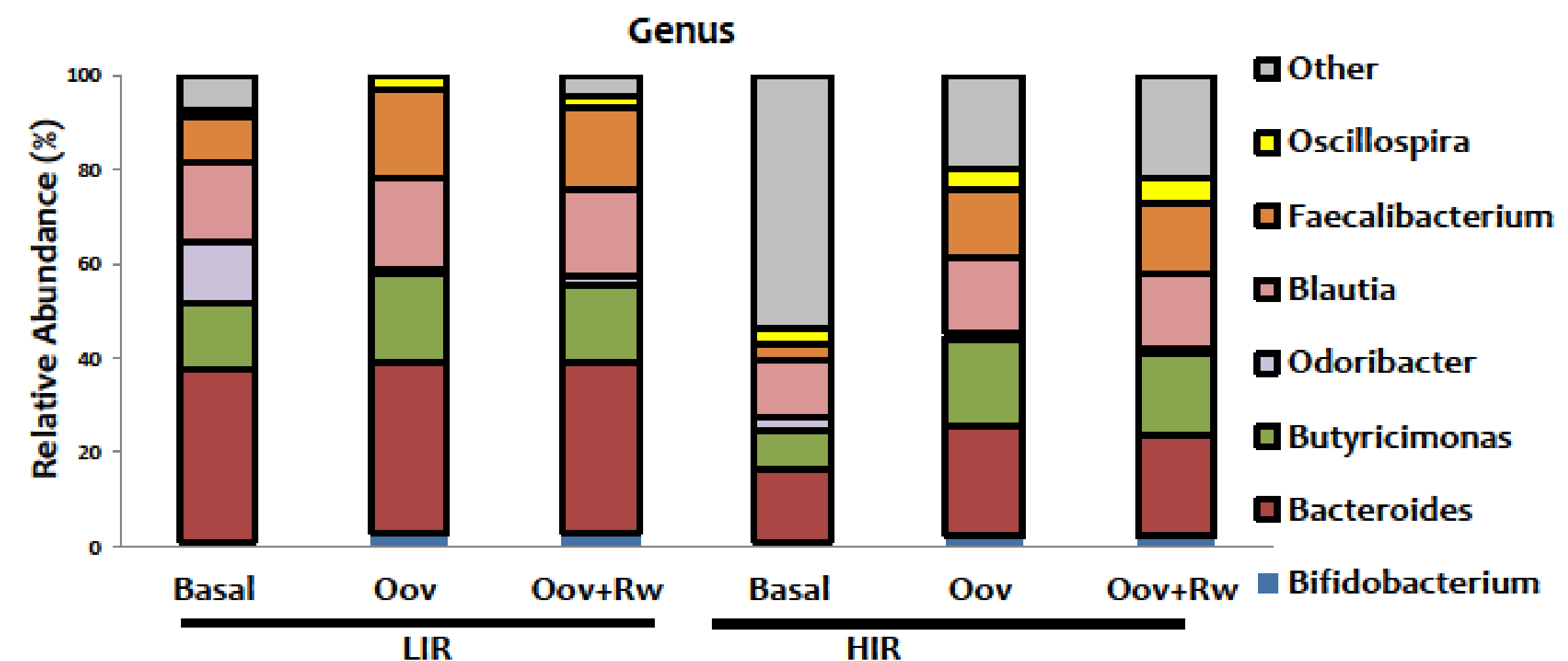

